Washington L. C. dos Santos · Jameel Rahman

Nigel Klein · David K. Male

\title{
Distribution and analysis of surface charge on brain endothelium in vitro and in situ
}

\begin{abstract}
Vascular endothelial cells are associated with a number of anionic molecules. These anions are important in endothelial function, particularly in regulating permeability, haemostasis and cellular traffic. To explore the nature and distribution of anions on the brain endothelial cell (BEC) surface, we have examined rat brain endothelium in culture, and in situ. The anionic sites were probed with cationic colloidal gold and cationised ferritin, and visualised by light microscopy. Additionally we compared the distribution of the anionic sites on BEC with that present on other endothelial cell types in culture. The predominant anion detected on BEC was heparan sulphate (HS). This was distributed throughout the cell membrane, but was most densely associated with intercellular junctions. This pattern was distinct from the anionic locations observed in endothelia from aorta and epididymal fat microvessels. The distribution of anions was dependent on the age of cultured cells, with only minimal levels of HS seen at the periphery of younger cells. The nature and distribution of negative charge was different in situ. Here, sialic acid was the major surface anion, with only a small contribution from HS. The significance of these findings are discussed in relation to endothelial function in normal tissue and in pathological conditions.
\end{abstract}

Key words Endothelium - Negative charge . Glycosaminoglycans $\cdot$ Heparan sulfate $\cdot$ Sialic acid

W. L. C. dos Santos (《) - J. Rahman - D. K. Male Institute of Psychiatry, Department of Neuropathology, De Crespigny Park, Denmark Hill, London SE5 8AF, UK Tel.: 44-071-7035411; Fax: 44-071-7083895; email:w.dossantos@ucl.ac.uk

N. Klein

Molecular Immunology Unit, Institute of Child Health, London, UK

\section{Introduction}

The negative charge associated with endothelial cells (EC) is considered to be an important determinant of vascular endothelial function. Endothelial anions are thought to be particularly involved in regulating the transit of proteins and cells from the circulation, in preventing mural thrombus formation and in locating cytokines and growth factors at the endothelial surface $[2,7,8,25-28]$.

The role of endothelial anions in human disease has yet to be fully determined. It is clear, however, that negatively charged sites are extremely sensitive to both biochemical stimuli, such as inflammatory cytokines $[10,12]$, and physiological stimuli such as blood pressure elevation [19]. Furthermore, there is now evidence that in certain pathological conditions, alteration in endothelial anions may be an important component of the disease process $[5,18]$.

Brain endothelium is morphologically and functionally distinct from other EC. They possess continuous tight junctions and a paucity of endocytotic vesicles $[1,9]$ which helps maintain the highly regulated environment found in cerebral tissue. In both the normal state and following activation by inflammatory cytokines, leukocyte adhesion and transendothelial migration is low in comparison with EC from other sites $[13,23]$. Some of the unique characteristics of brain EC (BEC) in vivo, are maintained in culture $[1,9]$.

Previous studies on brain endothelium in situ $[19,20$, $24,29]$ or in culture [30] have shown a regular distribution of the anionic sites all over the plasma membrane, with higher density on the apical surface of the EC [29]. A similar appearance of the negative charge has been described on other continuous microcirculation [26] and on human umbilical vein EC (HUVEC) in culture [10], when preserved by aldehydic fixation. In some microvascular beds, fenestrations and vesicles are associated with higher density and particular constitution of the negative charge $[3,4,25,26]$. Recently microdomains, mainly associated with heparan sulphate (HS), were identified also on HUVEC when the anionic sites were preserved by alcoholic fixation $[10,11]$. 
In this study we have characterised the distribution and nature of the anionic sites on the surface of BEC in culture and in situ. We have also compared BEC with other EC, derived from aorta, retina, and epididymal fat microvessels. These experiments may shed light on the role played by endothelial anions in regulating the morphological and functional properties of the blood-brain barrier (BBB).

\section{Materials and methods}

\section{EC monolayers}

Brain microvascular endothelia were isolated from 3-month-old inbred female Lewis rats [9]. Cerebral cortex obtained by dissection, was chopped and digested for $1 \mathrm{~h}$ at $37^{\circ} \mathrm{C}$ in $15 \mathrm{ml} 0.1 \%(\mathrm{w} / \mathrm{v}) \mathrm{col}-$ lagenase/dispase (Boehringer), $10 \mu \mathrm{g} / \mathrm{ml}$ DNase (Sigma), and 0.147 $\mu \mathrm{g} / \mathrm{ml} N$-p-tosyl-L-lysine chloromethyl ketone (TLCK, Sigma) in $\mathrm{Ca}^{2+}$-and $\mathrm{Mg}^{2+}$-free balanced salt solution (BSS, Gibco), containing $10 \mathrm{mM}$ HEPES, $100 \mathrm{U} / \mathrm{ml}$ penicillin and $100 \mu \mathrm{g} / \mathrm{ml}$ streptomycin. Tissue was further disrupted by aspiration through a narrow-bore pasteur pipette, and myelin removed by density fractionation in 250 $\mathrm{mg} / \mathrm{ml}$ BSA centrifuged at $1000 \mathrm{~g}$ for $20 \mathrm{~min}$. The pellet was digested for a further $3 \mathrm{~h}$, at $37^{\circ} \mathrm{C}$, in $5 \mathrm{ml}$ of the collagenase/dispase solution, containig DNase and TLCK (as above), resuspended in BSS, and separated from contaminating cells by centrifugation for 10 min on Percoll gradients, at $1000 \mathrm{~g}$. (Gradients were established by centrifugation of $50 \%$ isotonic percoll at $25000 \mathrm{~g}$ for $1 \mathrm{~h}$.)

Aortic endothelia were obtained by placing small pieces (2-3 $\mathrm{mm}^{2}$ ) of dissected rat aorta, luminal-side down on collagen-coated plates, in D-valine cell growth medium [MEM-D valine (Gibco) containing $10 \% \mathrm{FCS}, 75 \mu \mathrm{g} / \mathrm{ml}$ endothelial growth supplement (ECGS, Sigma), $40 \mu \mathrm{g} / \mathrm{ml}$ heparin, $2 \mathrm{mM}$ glutamine, $100 \mathrm{U} / \mathrm{ml}$ penicillin and $100 \mu \mathrm{g} / \mathrm{ml}$ streptomycin]. After 3 days the explants were removed and the outgrowing EC expanded. They were passaged by trypsinisation when confluent [16].

Epididymal fat endothelium was isolated by dissecting the fat pad from the capsule of male Lewis rats. A piece of approximately $1 \mathrm{~cm}^{2}$ was finely chopped with scissors and digested for $1 \mathrm{~h}$ at $37^{\circ} \mathrm{C}$ in collagenase/dispase/DNase/TLCK as above. The digest was triturated and filtered through a sterile nylon mesh of 0.45 $\mathrm{mm}$. The filtered suspension was then washed before plating out [23]. Cultures of retinal endothelium were kindly provided by $\mathrm{Dr}$. John Greenwood (Institute of Ophthalmology, London, UK).

Brain, retinal, aortic and epididymal fat EC were cultured in 50-ml tissue culture flasks, or plastic culture chamber slides, previously coated with collagen type I (Sigma), in Ham's F-10 medium (Gibco) containing $75 \mu \mathrm{g} / \mathrm{ml}$ ECGS, $40 \mu \mathrm{g} / \mathrm{ml}$ heparin, 2 $\mathrm{mM}$ glutamine, antibiotics and $20 \%(\mathrm{v} / \mathrm{v})$ bovine plasma-derived serum. The cultures were maintained at $37^{\circ} \mathrm{C}$ in $5 \% \mathrm{CO}_{2}$, and fed every 2 days until they had formed confluent monolayers. Details on the characterisation of the different endothelia are presented elsewhere $[1,6,9,16]$.

\section{Brain tissue preparation for histochemistry}

Fragments of brain tissue, isolated from six inbred female Lewis rats, were sliced $3 \mathrm{~mm}$ thick and fixed in $2.5 \%$ glutaraldehyde at $4{ }^{\circ} \mathrm{C}$ for $1 \mathrm{~h}$ or in cold methanol. The methanol fixation was carried out by immersing the tissue in $-20^{\circ} \mathrm{C}$ methanol and leaving at $4^{\circ} \mathrm{C}$ overnight. The specimens were washed in BSS, transferred to $70 \%$ ethanol and embedded conventionally in paraffin. Sections ( $4 \mu \mathrm{m}$ thick) were dewaxed and stained with cationic colloidal gold (CCG).

\section{Identification of anionic sites on the EC}

Different fixatives (glutaraldehyde with or without tannic acid, osmium tetroxide, methanol, acid alcohol, formalin) were tested to determine their ability to preserve the anionic sites on the $\mathrm{BEC}$ surface in culture. At the light microscopy level, aldehydic and osmic fixation produced a similar pattern of staining which was different from that produced by alcoholic fixation. Therefore, most of the experiments were performed using methanol or glutaraldehyde fixation.

BEC cultures were washed four times with cold BSS, fixed with cold $\left(\leq-20^{\circ} \mathrm{C}\right)$ methanol, or $2 \%$ glutaraldehyde, for $15 \mathrm{~min}$, and washed with BSS. Cell cultures and tissue sections was preincubated with the buffer in which the staining were performed. They were then incubated with $1 / 100 \mathrm{CCG}(5-\mathrm{nm}$ particles, BioCell) solution in BSS (pH 1.2-2.0) or citrate/phosphate buffer ( $\mathrm{pH}$ $2.8-4.0$ ), for $1 \mathrm{~h}$, at room temperature ( $\mathrm{R}^{\prime} \mathrm{T}$ ), and washed three times in distilled $\mathrm{H}_{2} \mathrm{O}$. The reactions were visualised by enhancing with silver (Sigma) and counterstaining with Harris's haematoxylin $[10,11]$. As controls, the gold reagent was either omitted or mixed with $1 \mathrm{mg} / \mathrm{ml}$ dextran sulphate or neutral dextran (Sigma). Alternatively some specimens were preincubated with 1 $\mathrm{mg} / \mathrm{ml}$ poly-L-lysine.

To reveal the anionic sites at $\mathrm{pH} 7.4$, pre-fixed cultures or sections were incubated with $1 \mathrm{mg} / \mathrm{ml}$ cationised ferritin (CF), for $1 \mathrm{~h}$ at RT. They were washed, post-fixed with $2 \%$ glutaraldehyde in BSS pH 7.4 for $15 \mathrm{~min}$, and incubated with albumin conjugated with colloidal gold (5-nm particles). After a further wash they were once more fixed with $2 \%$ glutaraldehyde, in BSS $\mathrm{pH} 7.4$ for $15 \mathrm{~min}$. The staining was enhanced with silver and counterstained with Haris' haematoxylin. The purpose of the multiple fixation. with glutaraldehyde was to cross-link the ferritin or the gold-conjugated albumin, at the sites of reaction. Each step of aldehydic fixation was followed by quenching of free aldehyde groups with $50 \mathrm{mM}$ TRIS/ $\mathrm{HCl}, \mathrm{pH} 7.5$, for $30 \mathrm{~min}$ at RT. As a control for these experiments, CF was replaced by native ferritin (NF) in the same number of wells.

\section{Electron microscopy}

For electron microscopy, fragments of brain capillaries were seeded directly on collagen gels [22] or transferred from 50-ml flasks when nearly confluent.

Confluent cultures were washed with BSS, fixed with methanol and labelled with CCG (15-nm particles) using the same procedure as described above, but omitting the enhancing step. The cultures were then post-fixed in $2 \%$ glutaraldehyde in cacodylate buffer, $\mathrm{pH} 7.2$, for $1 \mathrm{~h}$ at RT, followed by $1 \%$ osmium tetroxide (20 min, RT), and embedded in Spur resin. Ultrathin sections were examined with an Hitachi EM-H600 electron microscope at $75 \mathrm{~kW}$.

\section{Characterisation of endothelial anionic sites}

Pre-fixed cultures of BEC or methanol-fixed brain sections were washed with BSS and incubated with either $10 \mathrm{U} / \mathrm{ml}$ heparinase III (EC 4.2.2.8), $1 \mathrm{U} / \mathrm{ml}$ chondroitinase ABC (EC 4.2.2.4), or $1 \mathrm{U} / \mathrm{ml}$ of neuraminidase type $X(E C$ 3.2.1.18), all from Sigma (UK), in BSS, for $4 \mathrm{~h}$ at $37^{\circ} \mathrm{C}$. The specificity of these enzymes, and their efficacy on fixed tissue was assessed as described previously [10, 11].

\section{Results}

Distribution of the anionic sites on $\mathrm{BEC}$

\section{Cultures}

Following glutaraldehyde fixation, cultures stained with $\mathrm{CCG}$ at $\mathrm{pH}$ 1.5-3.5 exhibited a dense layer of particles distributed uniformly over the plasma membrane (Fig. 1a, compare to Fig. 2b). The staining pattern was less intense and varied more between individual cells at lower $\mathrm{pH}$ 

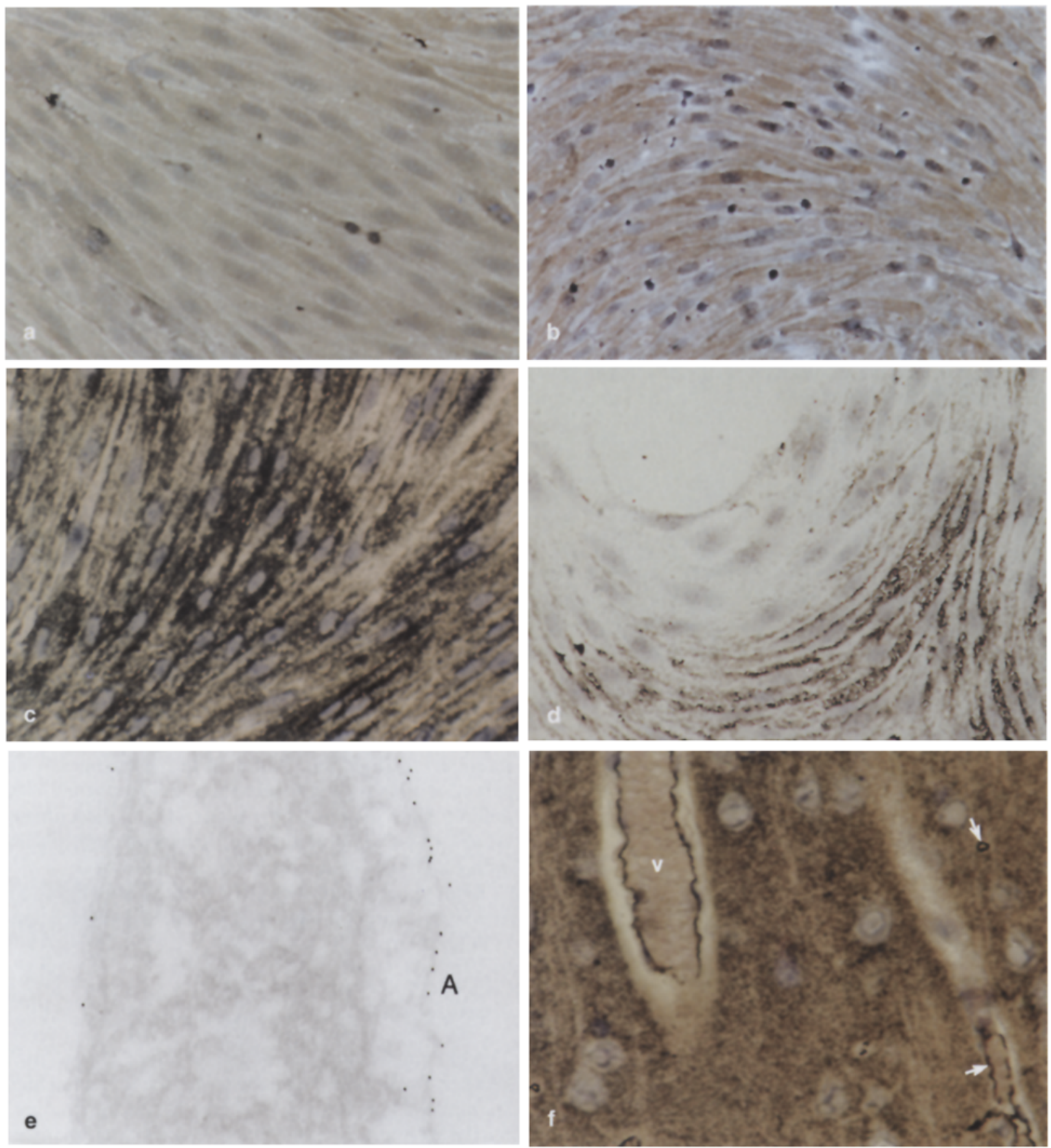

Fig. 1 a-e Negative charge on brain endothelial cells in culture and (f) in situ. a, b Glutaraldehyde fixation. a Uniform distribution of the anionic sites on the cells stained at $\mathrm{pH} 3.0$ with cationic colloidal gold (CCG), showing slight variation in intensity among the cells (compare with Fig. 2b). b Cultures, stained with cationised ferritin $(\mathrm{CF})$ at $\mathrm{pH} 7.4$, exhibit anionic sites distributed all over the plasmalemma, with a slight increase at the periphery of the cells. c-f Methanol fixation, and staining at $\mathrm{pH} 2.0$ with CCG. c Confluent cultures reveals distribution of anionic sites at the peripheral areas of the cells. d Non-confluent (5-day) culture. Anionic sites develop progressively on the central areas of the cell patches. e Transverse section of endothelial cell on electron microscopy (EM), showing anionic sites predominantly on the apical (A) surface of the cell. $f$ Section of brain cortex. Uniform distribution of the anionic sites on brain endothetial cells ( $V$ venule, arrows small blood vessels) a, c, d, f $\times 380 ; \mathbf{b} \times 190 ; \mathbf{e} \times 28000$

(1.5-2.0) values. The substrate, exposed by cell detachment, and some contaminant cells also stained intensely. At pH 7.4, using CF, the staining pattern was similar to that seen at low $\mathrm{pH}$. In some areas, however, particles were more densely deposited at cell borders (Fig. 1b)

The pattern of staining with CCG after methanol fixation was more dependent upon $\mathrm{pH}$. At $\mathrm{pH}$ 1.5-2.0 the anionic sites were predominantly located at the cell periphery, close to the cellular junctions (Fig. 1c). Sometimes a fine lattice-work could be seen extending across adjacent cells (Fig. 1d). This distribution of the anionic sites on $\mathrm{BEC}$ remained unchanged after the first subculture, and 
developed in cultures grown in medium which has not been suplemented with heparin.

At $\mathrm{pH}$ 3.0-4.0 a similar pattern was seen. However, additional staining was often seen in central areas of the cell membrane and over the entire plasma membrane in some areas of the culture.

Anionic sites were observed to be distributed all over the plasmalemmal membrane at $\mathrm{pH} 7.4$, with a slight to moderate increase in density at the periphery of the cells. Although the staining was more uniform than with CCG, some variability between cells was still present.

To verify the location of these anionic sites preserved by methanol fixation, some BEC cultures were stained and examined ultrastructurally. Gold particles decorated both the apical and basal surfaces of the EC membrane with a higher density of sites observed on the apical aspect of the plasmalema (Fig. 1e).

\section{Tissue sections}

The best staining results on tissue were obtained with methanol fixation. Formalin or glutaraldehyde caused some degree of shrinkage of the small blood vessels in the brain, interfering with the labelling of the luminal surface of the EC.

After methanol fixation, and staining at $\mathrm{pH} 2.0$ 3.0 , the vascular bed in the brain was the most evident structure observed (Fig. 1f). The gold particles were distributed uniformly on the EC from arterioles, capillaries and venules. In the larger blood vessels, especially those in the leptomeninges, the staining appeared to be slightly stronger on the arterial endothelium. The adventitia of the larger blood vessels and the medial aspect of some arterioles, presented discrete to moderate fibrilar staining. At lower $\mathrm{pH}(1.2-1.5)$ only slight staining was observed on the adventitia of arterioles and venules, and a rather strong staining was revealed on some neuronal cells.

On the specimens fixed by immersion in glutaraldehyde, venules, arterioles and capillaries stained irregularly, some exhibiting a linear uniform deposition of the gold particles on the endothelial surface, and a less-intense staining of the adventitia. Specimens from two animals fixed by perfusion, presented a more regular distribution of charge, similar to that observed with methanol fixation.

At pH 7.4 using CF, all cellular and non-cellular structures were stained. At this $\mathrm{pH}$ the intensity of staining on the EC was similar to that present on the surface of the other cell types.

In the control experiments, omitting $\mathrm{CCG}$ or mixing the reagent with dextran sulphate, or replacing CF by NF, resulted in no staining on the cell surface. In contrast, neutral dextran had no effect on staining. Preincubation of the cultures with poly-L-lysine completely disrupted the labelling seen with CCG and, in addition, increased the background indiscriminately over both cells and substrate.
Nature of the anionic sites on BEC

The staining patterns observed on BEC at various $\mathrm{pH}$ values reflects the dissociation characteristics of the different anionic groups. At lower $\mathrm{pH}$ values, highly acidic groups with a lower pK are selectively stained. To assess the contribution made by different anionic groups, we predigested the specimens with heparinase III, which specifically degrades HS and heparin, neuraminidase, which removes sialic acid, and chondroitinase (ABC), which removes chondroitin and dermatan sulphate [10].

\section{Cultures}

The anionic sites observed on the BEC at $\mathrm{pH} 1.5-3.0$ after methanol fixation, were almost completely abolished by treating the cells with heparinase III (Fig. 2a). The changes were more striking at the edges of the cells, where the fibrillary and particulate staining essentially disappeared. A sparse array of particles remained uniformly distributed on the plasmalemmal membrane, and was more evident at higher $\mathrm{pH}$ 3.0. The staining on the substrate, exposed by detachment of the cells, and on most of the contaminant cells remained unchanged. On the cultures fixed with glutaraldehyde, the staining was only slightly decreased by heparinase III.

Neuraminidase reduced most of the non-peripheral staining observed after methanol fixation, and virtually cleared the staining observed on BEC fixed with glutaraldehyde (Fig. 2b). Although these changes were observed at $\mathrm{pH} 2.0$, they were more evident at $\mathrm{pH} 3.0$. The anionic sites related to the areas of cell detachment and those present on some contaminant cells did not change with treatment by neuraminidase.

Using chondroitinase $\mathrm{ABC}$, there was a slight to moderate reduction of the staining on the cell surface and subcellular matrix.

\section{Tissue sections}

The enzymatic analysis of the negative charge in tissue sections was more dependent on the $\mathrm{pH}$ at which the staining was performed. At pH 1.2-1.5, the anionic sites on the adventitia of the blood vessels was abolished by heparinase III, and intensified by both neuraminidase or chondroitinase $\mathrm{ABC}$.

At pH 2.0 most of the negative charge present on surface of the endothelial cells in arterioles, capillaries and venules disappeared with treatment with neuraminidase (Fig. 2c). Heparinase III had minimal effect on degrading these anionic sites (Fig. 2d) and no change was observed with chondroitinase. Enzyme treatment prior to staining at $\mathrm{pH} 3.0$, revealed distinct anion patterns for the different vessels studied. While labelling was only slightly decreased in capillaries and venules after treatment with neuraminidase, anions associated with arterioles were almost abolished (Fig. 2e). Heparinase or chondroitinase had no effect on the staining at this $\mathrm{pH}$. 

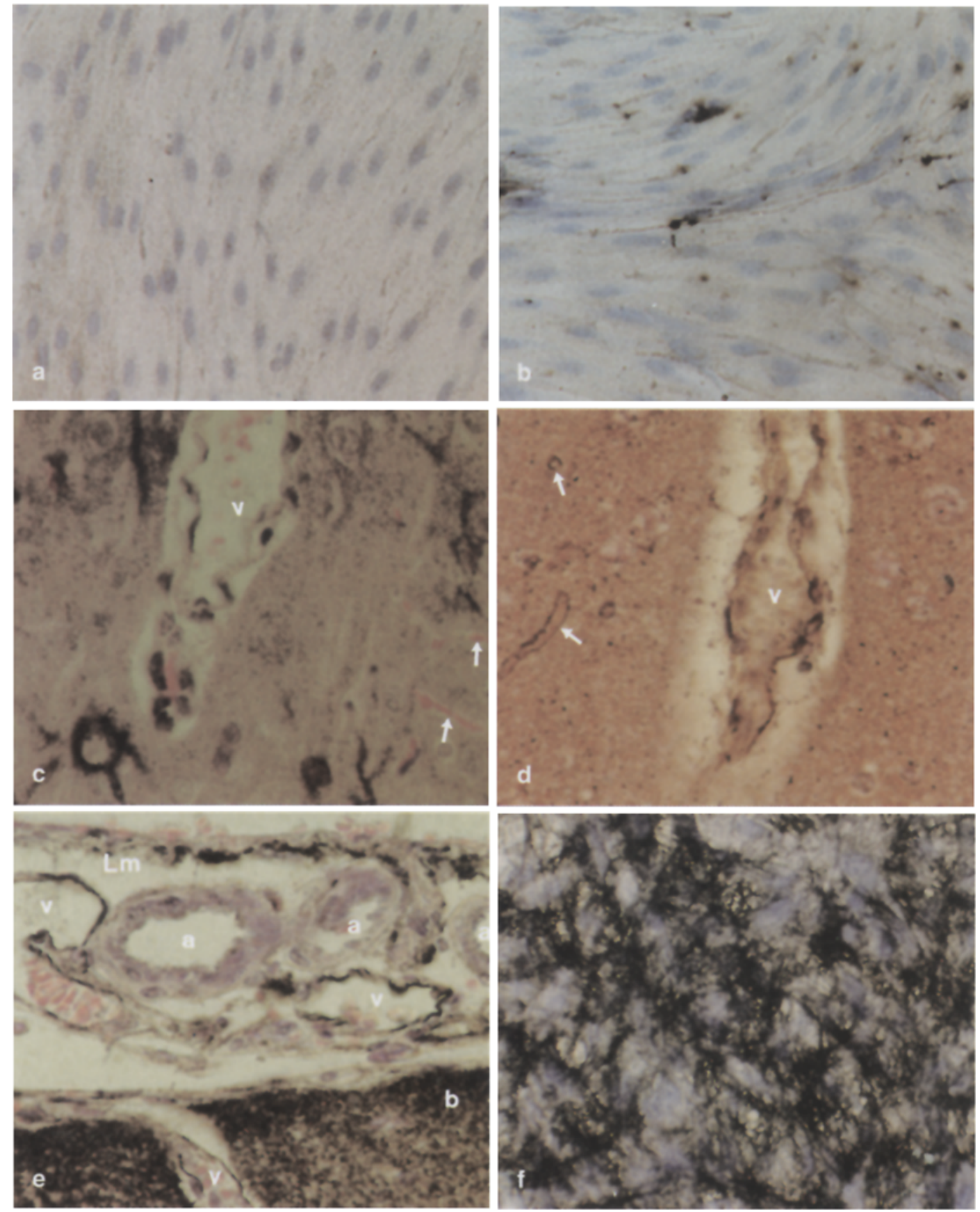

Fig. 2 Effect of enzyme treatment on the anions on BEC in culture $(\mathbf{a}, \mathbf{b})$ and in situ $(\mathbf{c}-\mathbf{e})$, both stained with CCG. a Most of the anionic sites revealed at $\mathrm{pH} 2.0$, after methanol fixation, are sensitive to heparinase III (compare with Fig. 1c). b Neuraminidase abolish almost completely the staining observed at pH 3.0 after fixation with glutaraldehyde (compare with Fig. 1a). c,d Anionic sites preserved with methanol and stained at $\mathrm{pH} 2.0$ ( $V$ venule, arrows small blood vessels). c Neuraminidase abolish most of the staining on BEC (compare with Fig. 1f), while, as shown in d, heparinase III has only minimal effect. e At $\mathrm{pH} 3.0$ neuraminidase reduces substantially the staining on arterioles, the staining on venules is less affected ( $a$ arteriole, $b$ brain cortex, $L m$ leptomeninges, $v$ venule). f Aortic endothelium in culture: anionic sites distribute as a fibrilar network. a-f $\times \mathbf{3 8 0}$

No reduction on CF staining at $\mathrm{pH} 7.4$ was observed in the cultures or in tissue sections, after treatment with any of these three enzymes.

The effect of culture duration on CCG staining

The intensity and distribution of negative charge on BEC was found to be age dependent. During the initial experiments, using confluent monolayers, we observed some variability in the intensity of staining at different areas of the cultures fixed with methanol and stained at $\mathrm{pH}$ 1.5-4.0. To see whether such variability was determined by the population of EC from different segments of brain 
microcirculation, or if it was due to different phases of the cell cycle, we decided to follow the development of surface charge of BEC in culture. The cultures were then stained from day 3 , when the cells start to spread from the fragments of blood vessels laid in culture, to day 19 , when they are confluent.

Although some staining was observed at days 3-4, it was always irregular and associated with the connective matrix associated to the capillaries laid in culture. The anionic sites on the EC were not detected until day 5 (Fig. 1d), and increased progressively to cover confluent areas of the culture. The intensity of staining was greatest in the older areas of the confluent cultures. Even in these confluent cultures, new areas of growth showed only minimal staining.

\section{Comparison of the anionic sites on BEC and other EC}

Using methanol fixation and CCG staining at $\mathrm{pH}$ 1.5-2.0, a similar pattern of anionic sites were observed with retinal endothelium. However, the patterns were completely different in aortic or epididymal fat endothelium. In these cultures the staining was seen as an entangled mesh of fibres extending over the cell surface (Fig. 2f).

\section{Discussion}

EC surface negative charge has been the subject of many studies using transmission electron microscopy on tissue preserved by aldehydic fixation. In this study we have used a technique developed for visualisation of anionic sites by light microscopy, with some adaptations for labelling tissue. The combination of alcohol or aldehyde fixation, staining in a wide $\mathrm{pH}$ range, and enzyme digestion enabled differences in the nature and organization of anions between cultures of different endothelia to be detected.

We demonstrate here that the pattern of anionic sites present on BEC and retinal endothelium was distinct, differing markedly from that observed on the aortic or the epididymal fat endothelium cultured under the same conditions. BEC anionic distribution was also different from the extensive network of fibrils reported to be associated with HUVEC [10, 11].

Intercellular junctions are important areas for communication between EC and for modulating the exchanges between blood and tissue. One of the characteristics of EC that constitute the BBB, is their ability to form continuous tight junctions $[1,9]$. In this study we found that the major distinction between BEC anion distribution and that of other endothelia was the high density of HS close to the intercellular junctions in BEC. Previous studies have noted that when the distribution of negative charge on EC was found to differ between organs, or within vascular segments of the same organ $[3,4,25,26]$, it was associated with functional specialisations of the cell such as fenestrations or vesicles. It is possible that the high density of negative charge near intercellular junctions observed in this study on BEC and retinal endothelium, may represent such a specialisation. HS proteoglycans are involved in cell-cell signalling and adhesion, tissue organization and cell migration $[7,27,28]$ and their intercellular position suggests that they are optimally located for the specialised functions and structure of the BBB. Further functional and morphological studies are required to determine the role of these glycosammoglycans (GAG) in relation to the constitution or maintenance of the $\mathrm{BBB}$ in culture.

Although the distribution of surface anions seen in BEC was distinct from other cultured EC, the nature of these sites was similar to that reported for other EC studied in vitro $[10-12,31]$. We found that like aortic monolayers $[12,21,31]$ and HUVEC $[10,11,22]$, HS was a major anion associated with cultured BEC.

In contrast, however, the contribution of HS to the negative charge of BEC in situ was usually less than that contributed by sialic acid. Similar findings has been reported in studies on bone marrow sinusoids [3] and brain microcirculation [29]. Simionescu et al. [25, 26] has also observed that neuraminidase reduced the number of anionic sites on pancreatic endothelium and muscle vasculature to a greater extent than with heparinase. However, as the staining in these studies was performed at neutral $\mathrm{pH}$ their relationship to our findings is unclear. Under similar $\mathrm{pH}$ conditions, we were unable to identify any effect of theses enzymes on the anion staining in either tissues or cultures. Similar results were obtained by Bruyn et al. [3] in bone marrow sinusoids, indicating that many other molecular groups associated with the cell membrane, will be negatively charged at physiological $\mathrm{pH}$ and may, therefore, be of functional significance.

The factors involved in determining the anionic composition of BEC are not currently known and may be relevant to understanding the anionic differences that exist between BEC in culture and in situ. One possible explanation is that the stage of cellular differentiation may be important in anion regulation. We observed that the maturity of cells in culture had a profound influence on the composition and distribution of surface anions. The amount of HS on young, developing cells was much lower than that seen on established monolayers. The growing regions of the cellular islands within the culture were particularly sparse in HS. In contrast, sialic acid residues were uniformly distributed in cells of all stages of development. This concurs with other studies which have also demonstrated that HS increases as cells grow to confluence. Indeed, HS-proteoglycan is the principal proteoglycan synthesised by confluent aortic EC monolayers [31], and HS represent 70-90\% of total GAG in cell surface fractions from confluent aortic endothelium in culture [12].

While the functional significance of cell surface anionic composition is currently speculative, many studies have highlighted a relationship between changes in surface charge and cellular maturation [14], development $[17,26]$, and malignancy [15]. In this study we have described the anionic properties of BEC in situ and in cul- 
ture, which may provide clues to their function in vivo. Future studies, using the approach described here, should help to define further the role of endothelial anions in $\mathrm{BBB}$ development and function.

Acknowledgements This work was supported by the Brazilian Research Council (CNPq) and the Multiple Sclerosis Society of Great Britain.

\section{References}

1. Abbott NJ, Hughes CCW, Revest PA, Greenwood J (1992) Development and characterization of rat brain capillary endothelial culture: towards an in vitro blood-brain barrier. J Cell Sci 103:23-37

2. Bradfield JWB, Born GVR (1969) Inhibition of lymphocyte recirculation by heparin. Nature 222:1183-1184

3. Bruyn PPH, Michelson S, Becker RP (1978) Nonrandom distribution of sialic acid over the cell surface of bristle-coated endocytic vesicles of the sinusoidal endothelium cells. J Cell Biol 78:379-389

4. Bush MS, Allt G (1990) Blood-nerve barrier: distribution of anionic sites on the endothelial plasma membrane and basal lamina. Brain Res 535:181-188

5. Gotloib L, Shostak A, Galdi P, Jaichenko J, Fudin R (1992) Loss of microvaccular negative charges accompanied by interstitial edema in septic rats' heart. Circ Shock 36:45-56

6. Greenwood J (1992) Characterization of a rat retinal endothelial cell culture and expression of $\mathrm{P}$-glycoprotein in brain and retinal endothelium in vitro. J Neuroimmunol 39:123-132

7. Hardingham TE, Fosang AJ (1992) Proteoglycans: many forms and many functions. FASEB $J 6: 861-870$

8. Hart MN, VanDyk LF, Moore SA, Shasby DM, Cancilla PA (1987) Differential opening of the brain endothelial barrier following neutralization of the endothelial luminal anionic charge in vitro. J Neuropathol Exp Neurol 46:141-153

9. Hughes CCW, Lantos PL (1986) Brain capillary endothelial cells in vitro lack surface IgG Fc receptors. Neurosci Lett 68: $100-106$

10. Klein NJ, Shennan GI, Heyderman RS, Levin M (1992) Alteration in glycosaminoglycan metabolism and surface charge on human umbilical cells induced by cytokines, endotoxin and neutrophils. J Cell Sci 102:821-832

11. Klein NJ, Shennan GI, Heyderman RS, Levin M (1993) Detection of glycosaminoglycan on the surface of human umbilical vein cells using gold conjugated poly-L-lysine with silver enhancement. Histochem J 25:291-298

12. Kobayashi M, Shumada K, Ozawa T (1990) Human recombinant interleukin $1 \beta$ and tumor necrosis factor a mediated suppression of heparin-like compounds on cultured porcine aortic endothelial cells. J Cell Physiol 144:383-389

13. Male D, Pryce G, Rahman J (1990) Comparison of the immunologic properties of rat cerebral and aortic endothelium. J Neuroimmunol 30:161-168
14. Marikovsky Y, Danon D (1969) Electron microscope analysis of young and old red blood cells stained with colloidal iron for surface charge evaluation. J Cell Biol 43:1-7

15. Marikovsky Y, Shlomai Z, Asher O, Lotan R, Ben-Bassat H (1986) Distribution and modulation of surface charges of cells from leukaemia-lymphoma lines at various stages of differentiation. Cancer 58:2218-2223

16. McGuire PG, Orkin RW (1987) Isolation of rat aortic endothelial cells by primary explant techniques and their phenotypic modulation by defined substrata. Lab Invest 57:94-105

17. Mills AN, Haworth SG (1991) Greater permeability of the neonatal lung. Thorac Cardiovasc Surg 101:909-916

18. Murch SH, MacDonald TT, Walker-Smith JA, Lavin M, Lionetti P, Klein NJ (1993) Disruption of sulphated glycosaminoglycans in intestinal inflammation. Lancet 341:711714

19. Nag S (1984) Cerebral endothelial surface charge in hypertension. Acta Neuropathol (Berl) 63:276-281

20. Nagy Z, Peters H, Huttner I (1983) Charge-related alterations of the cerebral endothelium. Lab Invest 49:662-671

21. Oohira A, Wight TN, Bornstein P (1983) Sulfated proteoglycans synthesized by vascular endothelial cells. J Biol Chem 258:2014-2021

22. Pawlowski N, Kaplan G, Abraham E, Cohn ZA (1988) The selective binding and transmigration of monocytes through the junctional complexes of human endothelium. J Exp Med 168: $1865-1882$

23. Pryce G, Santos W, Male D (1994) An assay for the analysis of lymphocyte migration across cerebral endothelium in vitro. $\mathbf{J}$ Immunol Methods 167:55-63

24. Schmidley JW, Wissig SL (1986) Anionic sites on the luminal surface of fenestrated and continuous capillaries of the CNS. Brain Res 363:265-271

25. Simionescu M, Simionescu N, Silbert JE, Palade GE (1981) Differentiated microdomains on the luminal surface of capillary endothelium. II. Partial characterization of their anionic sites. J Cell Biol, 90:614-621

26. Simionescu M, Simionescu N, Santoro F, Palade GE (1985) Differentiated microdomains of the luminal plasmalemma of murine muscle capillaries: segmental variations in young and old animals. J Cell Biol 100:1396-407

27. Tanaka Y, Adams DH, Hubscher S, Hirano H, Siebenlist U, Shaw S (1993) T-cell adhesion induced by proteoglycan-immobilized cytokine MIP-1 $\beta$. Nature 361:79-82

28. Tanaka Y, Adams DH, Shaw S (1993) Proteoglycans on endothelial cells present adhesion-inducing cytokines to leukocytes. Immunol Today 14:111-115

29. Vorbrodt AW (1987) Demonstration of anionic sites on the luminal and abluminal fronts of endothelial cells with poly-L-lysine-gold complex. J Histochem Cytochem 35:1261-1266

30. Vorbrodt AW, Trowbridge RS (1991) Ultracytochemical characteristics of cultured sheep brain microvascular endothelial cells. J Histochem Cytochem 39:1555-1563

31. Wight TS, Kinsella MG, Lark MW, Potter-Perigo S (1986) Vascular cell proteoglycans: evidence for metabolic modulation. Ciba Foundation Sym 124:241-259 